\title{
Development of QR-code based Interactive Dynamic Billboard System with Motion Detection
}

\author{
Yvonne Iradukunda ${ }^{1}$, Innocent Ciza $^{2}$, Salama Ndayisaba ${ }^{3}$, Devotha Nyambo ${ }^{4}$, Kisangiri Francis \\ Michael $^{5}$ \\ Research Scholar ${ }^{1-3}$, Lecturer ${ }^{4}$, Senior Lecturer ${ }^{5}$ \\ School of Computational and Communication Sciences and Engineering
}

The Nelson Mandela African Institution of Science and Technology, Arusha-Tanzania

\begin{abstract}
This paper presents the design and implementation of an intelligent Dynamic electronic billboard based on QR-Code (Quick Response Code) that can be used in a variety of interior locations such as offices, malls, universities, supermarkets, and other similar establishments. A screen display, a sensor such as a PIR motion sensor, and a QR-Code reader are all included in the system. Once scanned, the QR-Code should provide rapid access to information. While the motion sensor detects a nearby person and instructs the system to display the QR-Code on the billboard for the person to scan, the display device continues to display the company's products or announcements. The QR-Code contains Uniform Resource Locators (URLs) in our prototype, and once scanned, a user follows the link and begins exploring what he or she needs to see on a display device based on what the companies sell or communicate with the general public. As a result, the system allows users to interact with it by allowing them to search for what they need using QR-Codes. The Raspberry Pi houses the sensors and display device, allowing the system to relay sensory data to a web server using the HTTP (Hypertext Transfer Protocol) protocol. Because of its low cost, efficiency, and ease of use, the designed system is beneficial.
\end{abstract}

Key Words: Smart Advertisement, Dynamic Billboard, QR-Code, PIR Motion Sensor, Web Application, Raspberry Pi.

\section{INTRODUCTION}

Marketing is a crucial strategy for businesses to express commercial products to customers or establish product care in the thoughts of customers in order to improve brand positioning and visibility [1] . To be successful, information must be delivered to the appropriate people at the right time and in the right place [2]; according to Boundless Business [3] , billboards are a great way to share the information with consumers. A billboard is a large outdoor advertising structure that can be seen in the form of generally positioned in high-traffic places such as alongside significant highways in the United Kingdom and many other countries of the world [4]. Passing pedestrians and automobiles are exposed to massive advertisements on billboards. Users employ static and dynamic billboards to communicate information to clients. Banners are static billboards that can be found in offices, restaurants, shopping malls, bus and train stations, universities, airports, and other public and private locations.

Electronic information is shown on dynamic billboards, making it simple to target a specific audience [5].They are also used within firms or organizations to remind and inform customers about the company's policies and the project that needs to be completed (give them the news) [6], Universities use banners, direct mail, radio advertisements, magazines, emails, text messages, blogs, and websites to communicate information to staff and students. However, advertising through different means is more than just promoting, showing information and attracting customer's attention; Dynamic billboard advertising provides the ability to communicate with the system, providing more information about the advertisement and receiving feedback in real time. In this work, an interactive billboard which consists of parts such as Billboard, PIR sensor, Database and QR-Code is described. Those parts are interconnected and timely communicate together to deliver a complete working system which is called QR-Code based dynamic billboard. Email, website, magazine, banners and other static forms of communication lack the capacity to interact with customers by providing more information on offered products.

\section{RELATED WORKS}

Different researches were done and brought solutions to the challenges that institutions and businesses were facing during information transmission. Most institutions in EAC (East African Community), like universities and hospitals, are using banners 
that are not friendly environments, not targeting the potential customers, and most of them are holding expired information. Often, Dynamic billboards are rarely used to communicate the business product to the potential customer. Therefore, related works are divided into 2 main categories; there are billboards that are designed according to the technology used and others related to data mining but our project is mostly focused on technology used where our improved technology is easy and efficient. Recently developed billboards are embedded with high technology such as camera sensors that detect viewers using face recognition and provide relevant products to viewers thus minimizing waste of power and time; these technologies are expensive [7].

The customer, upon seeing an advertisement on display, may initiate a request from his mobile device to start an interactive communication process with Interactive Wireless Electronic Billboard [8]; however this system disturbs people since they are sending notification to everyone who is passing by. An IoT based Intelligent Billboard using Data Mining was developed, in this system the collected purchased information through RFID (Radio-frequency Identification) reader are stored into database, products that are stored in database are organized and categorized in a way that can be read by RFID. The products which are not yet sold out are also stored in a database with their detailed information so that special offers can be attached to them and increase their chance to be sold out. This billboard recommends the customers some products according to the customers' behaviour or preferences through data mining applied on sales then displayed on billboard [9]. This developed system is not real time and does not target customers individually; it displays the products from the server according to what is mostly purchased by others.

WSN (Wireless Sensor Networks) Based Smart Advertisement in intelligent Transportation System using Raspberry pi was implemented, this system is based on transportation system where billboard is placed on smart vehicle. When passengers are passing nearby the smart vehicle, the system sends notification to the clients through mobile phone to upload ads. The system has administration panel which facilitates a control of uploaded ads by legalizing and approve ads by checking which is eligible to be displayed before being sent to a server [10]. After approval and processing, ads are displayed on the billboard attached to the car. This system is targeting the advertiser not consumer because the ads are displayed according to the uploaded one not considering the viewers if they are in need of the product or not. The system administrator must be always there to approve the advertisements.

Smart Billboard System using data Mining and IoT implemented with considering both social and spatial data to provide promising results by considering user preference through giving them varieties of options to choose from. It delivers preferable sales to consumers, considering attention of the customers who are likely to buy the products based on buying patterns [11]. This system mainly focused on visualizing data. In Australia, the smart billboards connected with cameras were set up at all key locations to take the snapshot of the vehicles. The information such as the make, model and colour are then communicated from the database. From this data, targeted adverts would be shown with tag lines like "Hey black Merc driver, the heavens have opened" and a driver make note of it; this billboard displays where you can find the spare parts of the model of the passing Car [12]. The system has some drawbacks, because it is placed along the road it can cause accidents. Furthermore, the system is not interactive by giving users the option to communicate with it.

Generally, Existing systems are very programmed, and they display information according to the time set by the administrator, for example, the display displays information for no more than 15 seconds, which is insufficient for customers to absorb all information about the advertisement. This should be seen as amusing. The designed system solves this problem by allowing users to re-display advertisements and record all necessary information. Customers should be able to interact with the billboard and search for the products they require, as well as gather more information about those products. The customer may need products that are not their own and for which the face is not present to be categorized, as well as an option to interact with the billboard. If the billboard incorrectly categorizes age due to surgery performed by consumers to keep them looks young, that is the reason why they would need an option to interact with the display panel on their own.

A QR-Code based interactive billboard is discussed in this project; QR-Code technology was originally intended to track vehicles during the manufacturing process, but today, QR-Codes may be found in a variety of applications, such as product labelling and equipment identification [13]. Instead of printing several banners and papers at the expense of the company's resources, a QRCoded URL that connects clients to what service or products they need, decreases the size of information; by QR-Coded, URL links clients to what service or products they need. In this technology, QR-Codes are combined with dynamic billboards. The device uses a motion sensor to identify nearby people and interact with the system, allowing the QR-Code to be displayed on the billboard. The system then posts information about the current and upcoming activities of companies in a timely manner, reducing and saving resources used to print flyers and banners, and maintaining a clean and safe atmosphere.

\section{MATERIALS AND METHODS}

\subsection{System requirements}

Data was collected to gather the system requirements via an online questionnaire to the Nelson Mandela African Institution of Science and Technology(NM-AIST), School of COCSE (Computational and Communication Science and Engineering) and interview the business man whose company is Dynamic Billboard located in Tanzania-Arusha. 
The current way of conveying information to the public internally and externally is not efficient. In fact, according to the survey filled by students, most students don't visit the university website regularly and they are don't even check their email constantly. Therefore, an advertising billboard is needed to complement the current way of information.

The following are System Requirements:

\subsubsection{Functional requirements of this system}

$>$ To display relevant announcements

$>$ To have motion sensor to detect nearby person

$>$ To show a QR-Code that smart phone holders can scan to engage with the smart electronic billboard.

$>$ An administrator must be able to change data in the database through an interface.

\subsubsection{Non-functional requirements system}

Advertisement will be available in working hours.

$>$ Minimize system failures by writing resilient code that prevents and detects failures before they happen.

$>$ To ensure security of a billboard.

$>$ The technology must be easy to use and perform well in real time.

\subsection{Data Analysis}

The above information has given us the idea of developing an Indoor Billboard to complement the current advertisement system and announcements of events and other helpful communication. Existing billboards of Digital Dynamic Company are displaying the products of companies randomly. The use of indoor dynamic billboards is effective and has many advantages over the traditional approaches of advertising such as display products, giving nearby people to search what they need while some of the existing indoor billboards are still based on printed banners. It is then essential to work on a project that improves the current indoor advertising systems [6]. According to recent information smartphone users are more than three billion worldwide in 2021 [14]. Therefore, developing a system that helps smartphone users to take advantage of their device to get their desired information is necessary.

\subsection{Description of the System Design}

In this section, the figures below give an overview of the whole system. Figure 1 presents data flow of the system from the scratch and how it is displayed. The system is user-friendly which includes devices and websites. All data is stored in a database which by the end is viewed via a user-friendly interface. Each information is displayed in a given time of 30 seconds and replaced continuously by other information while figure 2 describes the interaction of the system with users. The system presents the interfaces which enable administrators to login and upload needed information into the system and perform other activities. To display QR-Code, smartphone users will scan a QR-Code to get the search bar through a web-page; the information searched is sent to the webserver and visualized at the billboard through the HTTP Communication protocol.

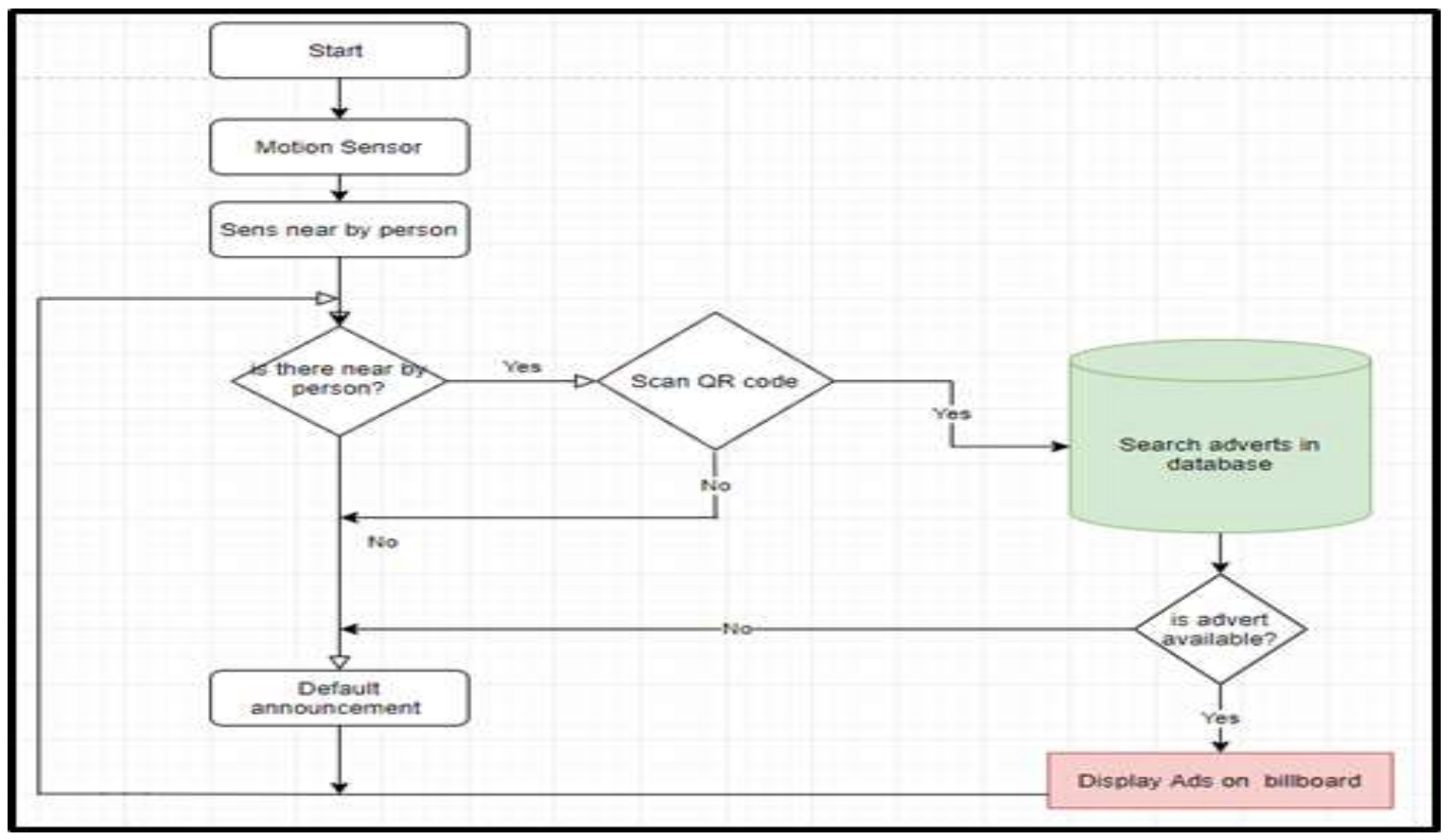

Figure 1: Flow chart diagram 


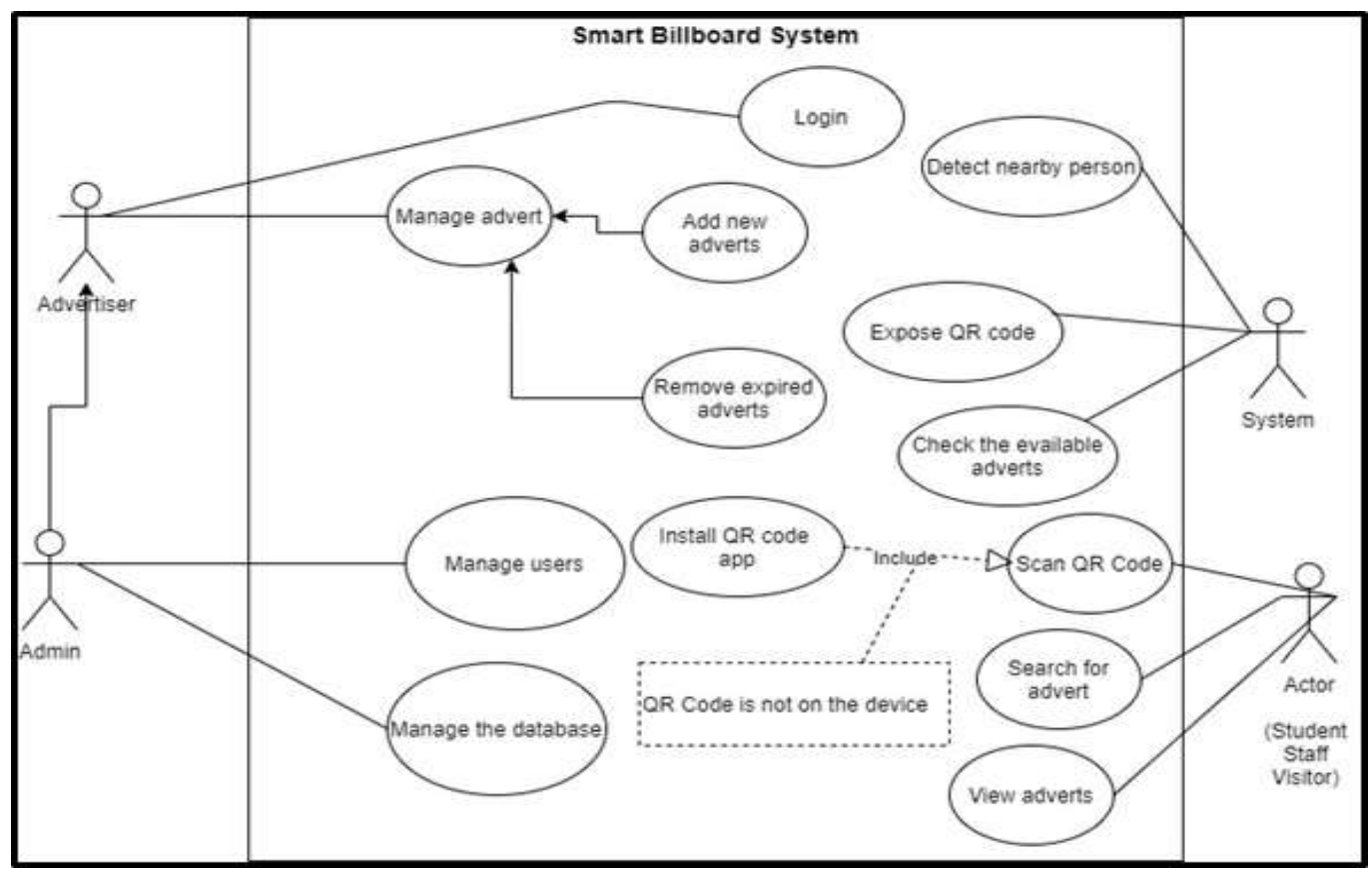

Figure 2: Use case diagram

\subsection{System Description}

\subsubsection{Hardware Description}

\section{a. Raspberry 3 model B+}

Raspberry Pi is a computer-like board with programmable GPIO (General Purpose Input Output) pins to enable the board to communicate with the external environment (Figure 3). A TV screen has been used as a screen and a USB (Universal Serial Bus) keyboard and mouse as peripheral input devices. An 8 GB Micro-SD card has been inserted in its appropriate place to enable the Raspberry Pi to load an Operating System, run programs and store data. The prototype uses Raspberry Pi 3 Model B+ is both the wireless network and wired network enabled. This feature allows the Raspberry Pi board to send sensed data to the web server through the HTTP Protocol. This board requires a 5.1V supply and can be powered through a USB peripheral with a Maximum total current draw of 1.2A [15].

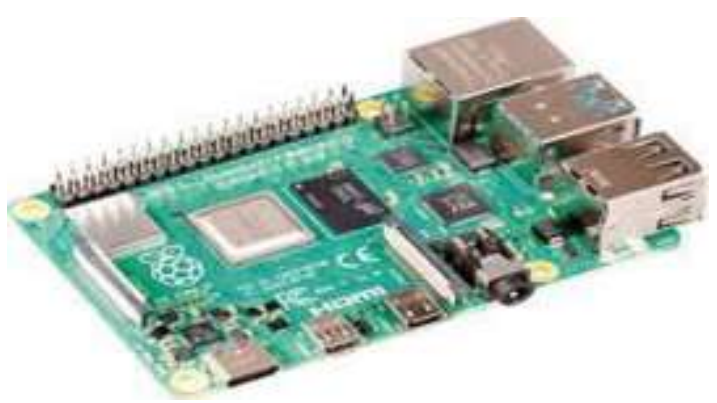

Figure 3: Raspberry Pi 3 Model B+ board

\section{b. PIR Motion Sensor}

PIR stands for Passive Infrared or Pyroelectric Infrared. The PIR sensor is one of the motion sensors. A motion sensor or motion detector is an electrical device designed to detect and measure the movement of people within its range of operation [16]. The PIR sensor used in our prototype can sense the motion of people at seven meters and an angle of less than $120^{\circ}$. This module was chosen to allow people to interact with the system even though they are a bite far away from the TV screen. This is powered directly from the raspberry pi board [17]. The PIR Motion Sensor used accepts a Power supply range of 5V-12V input voltage[18], [19]. It is directly powered from a raspberry pi board pin. 


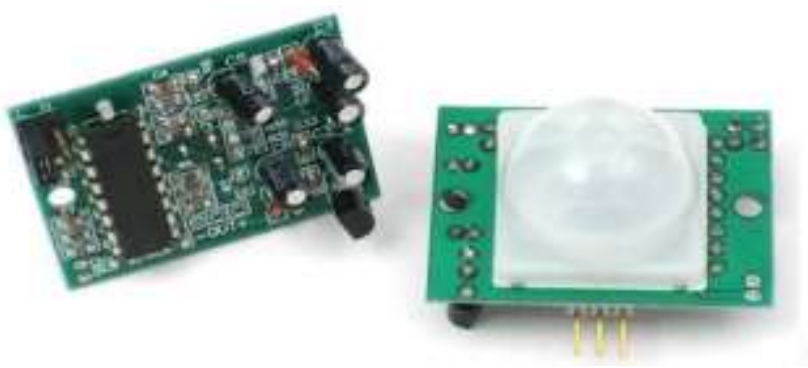

Figure 4: PIR Motion Sensor

\section{c. 32 inches-TV Screen}

For the developed prototype, the TV Screen acts as a billboard display. HDMI cable was used to connect the Raspberry Pi to the TV Screen. Power source and power cables are required for both the TV screen and Raspberry Pi. A 32 inches-TV Screen was used to visualize information in pictures and video formats. Therefore, files were converted into 720p (1366 X 768) pictures and videos resolution for better visualization.

\section{d. Computer}

During the software development, a computer with Linux Operating System was used for coding and testing. However, any computer can help access the web-based application to manage advertisements that are supposed to be displayed on the billboard. It must be connected to the internet to access the software application hosted on an online web server. Alternatively, a mobile device can be used to access the website either through a web browser (Mozilla Firefox) or a mobile web app that was created after converting the website to a mobile app through Appsgeyser.com, an online platform [20].

\section{e. Mobile phone Device}

Smartphones with suitable camera properties were used to scan the QR-Code. The owner must install any app (QR-Code Reader, Firefox web browser), which can help them scan the QR-Code and access the web-based application.

\subsubsection{Software description}

\section{a. Raspbian}

Raspbian is a free operating system that works on Raspberry Pi board. A Raspberry Pi Imager was used to download and install the Raspbian on a MicroSD card. After the operating system was written on the MicroSD card. The MicroSD card was inserted into the raspberry pi board. Once the Raspberry Pi was powered, it automatically loaded the Operating System [21].

\section{b. phpMyAdmin}

phpMyAdmin is free and open-source software that helps to manage databases through MySQL Database Management System. A prototype has one data and several tables, which helps record and manipulate all of the information regarding our web application. Our database is hosted on an online hosting provider which offers a phpMyAdmin tool to facilitate database management.

\section{c. Website}

A user-friendly website written in PHP, HTML, CSS, JavaScript (JQuery) and SQL(MySQL) was built to support administrative functionalities. The Sublime Text editor was used to write client and server-side scripts. Bitnami LAMP Stack 8.0.3-0 package was installed on Ubuntu distribution of Linux Operating system to enable the use of local web servers during the development and test of the web-based application. This package installed MySQL database and Apache Web-server modules on the computer. CodeIgniter and Bootstrap frameworks were used to develop an easy-to-maintain and responsive website quickly. A mobile web application was generated to facilitate access to our website from any mobile device which can install a mobile app file (APK file). However, a login (see Figure 8) is required for anyone who wants to access the system.

The software was successfully tested on Google Chrome (Version: 91.0.4472.88), Raspbian Chromium, Opera (Version:73.0.3856.344) and Mozilla Firefox (Version: 89.0) browsers.

\section{d. FileZilla Client for Linux}

It is a free FTP (File Transfer Protocol) solution and open-source software application for transferring files from the local server to the online server. The tool was used to upload local website to the online server. 


\subsection{Design of the prototype}

The following figures present the interconnection of devices used in system's architecture and circuits

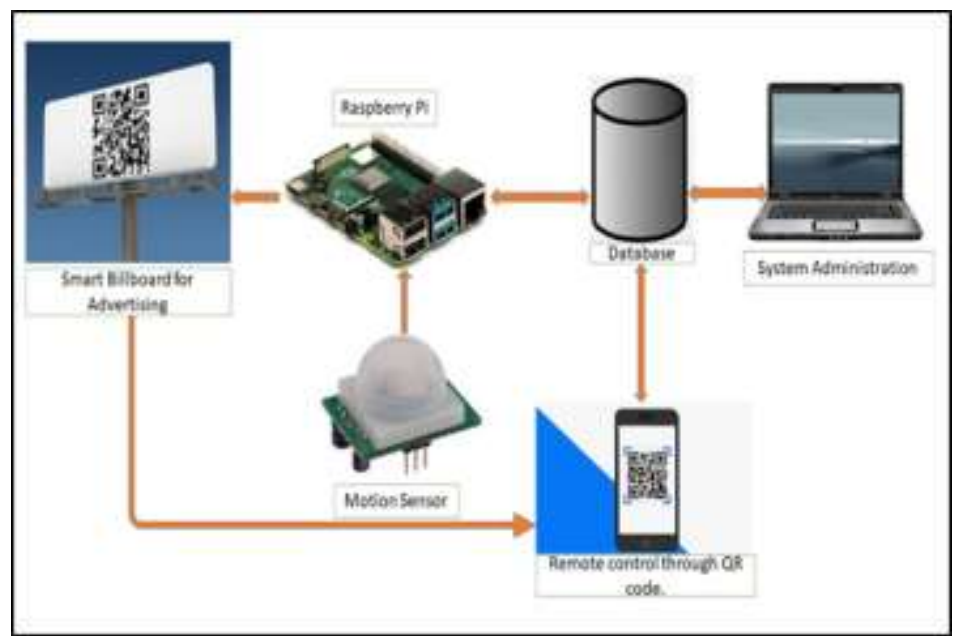

Figure 5: System architecture diagram

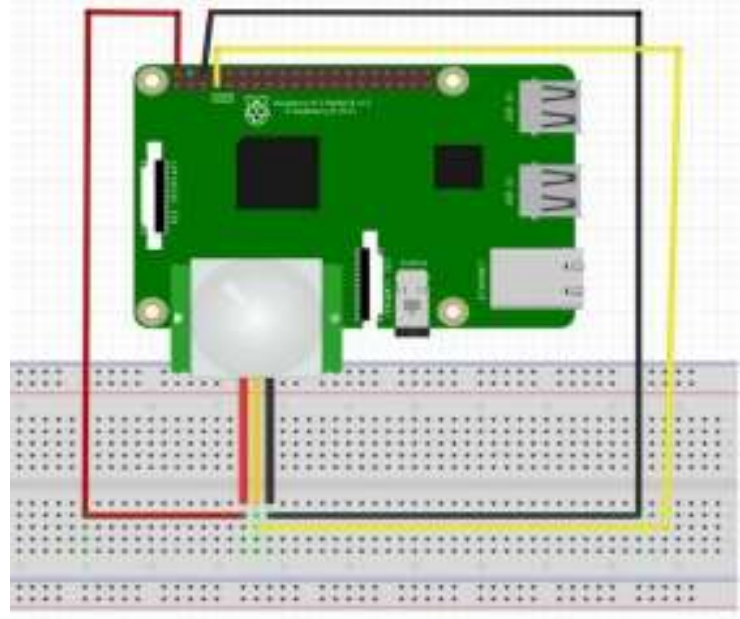

Figure 6: Circuit Diagram

\section{THE RESULTS AND DISCUSSION}

\subsection{Interactive billboard with QR-Code}

In this prototype, the designed system displays NM-AIST(The Nelson Mandela African Institution of Science and Technology) advertisements and announcements on a billboard that can be placed inside the university in front of the NM-AIST Amphitheatre, which is located near the main entrance and where staff and students pass by on their way to offices, library, conference hall, classes, restaurants, and laboratories; this is to complement to the university's current method of delivering announcements. Unlike a commercial billboard that was created to promote smartphones in universities [22], this billboard is not focused on academic issues.

The system developed by Rachwał(2019) [23], is a billboard that institutions use to recruit students and boost their recognition. It is used by universities such as the University of Poland, Michigan University, Concordia University, and other. This system uses banners that do not have the ability to change displays regarding the ongoing and upcoming events. Moreover, after advertising, banners must be removed, which is not environmentally friendly.

This prototype can be installed through the area where staff, students and visitors travel. When a user approaches the board, the system detects them and displays a QR-Code that the user may scan to find what they are looking for on the board. Academic announcements, the location of several essential places inside the university, forthcoming events, and extracurricular events are all featured on the billboard.

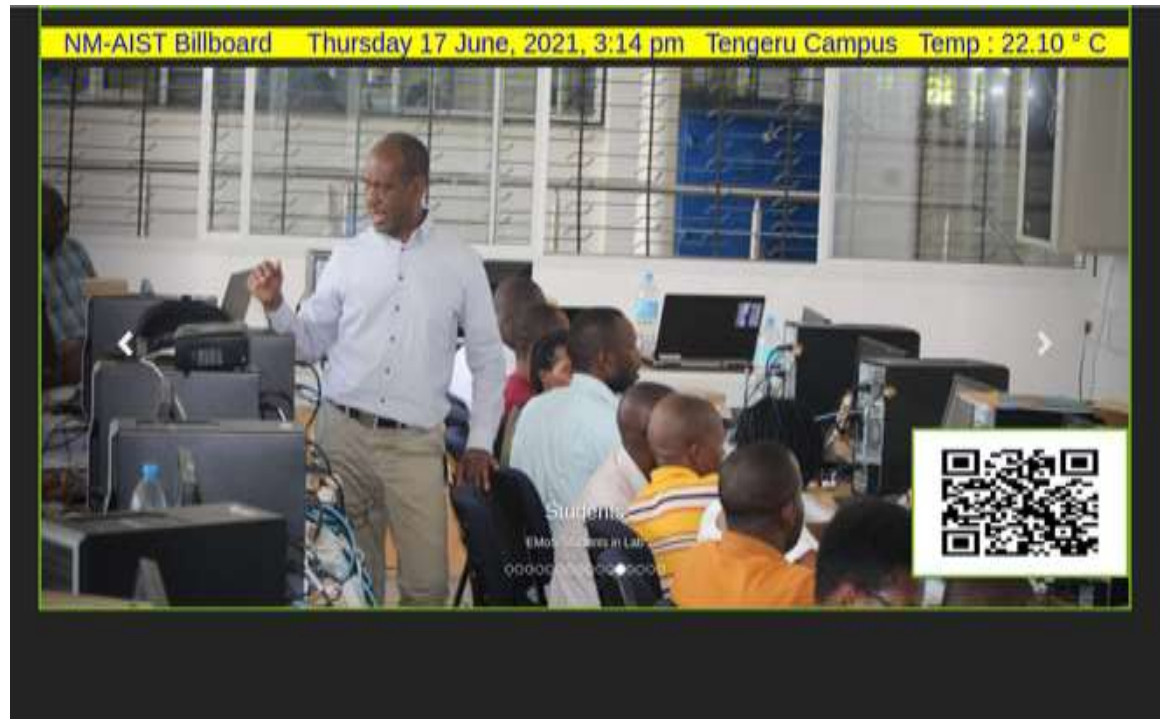

Figure 7: Billboard Screen 


\subsection{Improving user experience through mobile application}

A web mobile app was developed to facilitate the work of advertisement management as well as the search bar. The mobile app for advertisement management was able to record any kind of information in the form of pictures and videos as well. The advertisement recorded would be visualized on the billboard either randomly by the system itself or when the user scans the QRCode and search for it.

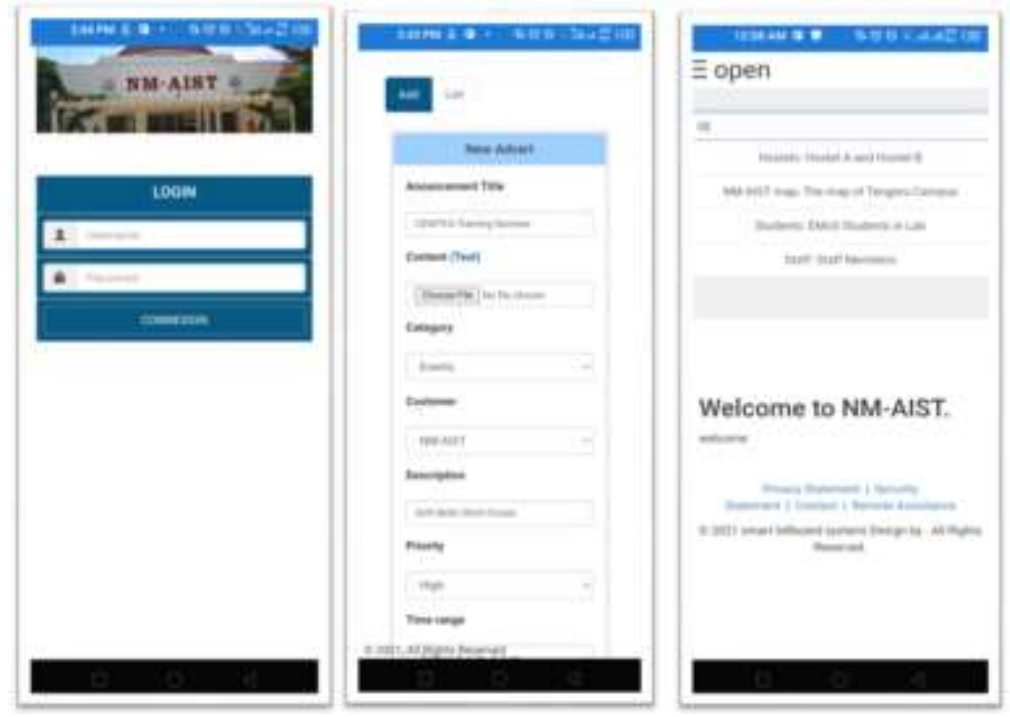

Figure 8: Left to Right; Login page, Adverts recording form, Adverts searching form

\subsection{Motion detection}

Motion detection detects human movement approaching a door, sounds an alarm, and turns on the camera to capture the face and match it to the owner's image [24]. This system may be installed everywhere in the house. A motion sensor can detect human movement, which is an advantage over an ultrasonic sensor, which can detect any moving item, including people or an object.

Each time a person approached, the human motion was examined. When motion is detected, the system shows a QR-Code in front of other moving advertisements, allowing users to engage with the system via the search feature. The billboard screen would show the search result.

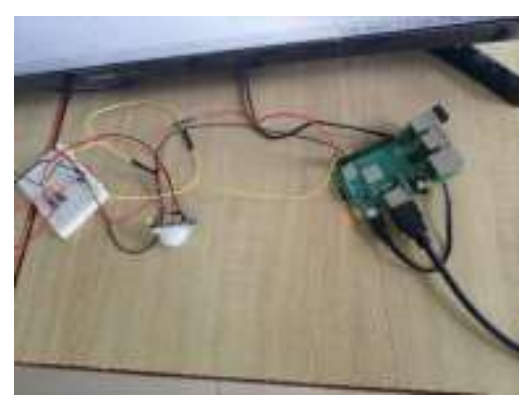

Figure 9: prototype of the system

The motion sensor and billboard were both connected to the Raspberry Pi and communicated through the board. The motion sensor detects nearby people and sends data to the board, which then sends the data to the cloud to be analysed. After 5 seconds, a QR-Code appeared on the screen to be scanned, which has a link interconnected with the billboard website and holds a lot of information about the institution, which is different from the existing billboard where some are static and others are dynamic, but displays the announcements imputed by administrators and controlled by themselves.

\subsection{Advantages of QR-Code based Interactive Dynamic Billboard with Motion Detection}

- It can be used to replace conventional methods of communicating information, such as static billboards, which are harmful to the environment.

- It enhances interactivity by allowing users to search for more information

- It gives users the option to re-display advertisements by capturing all relevant information.

- It can be installed indoors and so cannot cause an accident when positioned near a road. 
The project was developed and successfully tested using NM-AIST usual announcements. The Raspberry Pi was able to work more than 12 hours per day without either going to the sleeping mode or being turned off, which confirms the excellent performance of the prototype even when working in real applications. Billboard for advertisement through a web mobile based application enriched by the QR-Code is a better solution in advertisement systems. The companies as well as businesses would implement it to advertise products and services to their customers. The customers would then be able to check especially the needful information according to their convenience.

\subsection{Uniqueness of the developed prototype and testing results}

The proposed system is both interactive with nearby people and displays announcements; it can be used in a variety of institutions, including hospitals, to make appointments with doctors, display precautions to the public, and display available rooms for patients.

Scanning QR-Codes and searching for what they needed to present on the billboard screen has a lot of fun. This is a positive experience for advertisement systems since it improves the communication channel by allowing targeted customers to search for their needs.

\section{CONCLUSION AND RECOMMENDATION}

This prototype that improves advertising solutions through human motion detection and QR-Code was successfully designed and developed. Both Hardware and software work properly as it was expected.

The QR-Code based Interactive Billboard is suitable for many applications; as other indoor advertising billboards, it can be used in any institution or/and a business company that wants to eventually convey their information to the general public from their indoor places such as Supermarkets, Universities, Banks, Hospitals, and etc.

However, the implemented system can be upgraded in the future by adding more sensors such as cameras to recognize faces and identify whether a person is a staff or visitor and then customize announcements and advertising regarding the detected person. Moreover, applying machine learning algorithms is necessary to predict users' preference and taste in announcements considering what kind of information is mostly to be searched for. Finally, it is also essential to create a mobile app that can run on a bright Android TV Screen. This could decrease the activities performed by the Raspberry Pi and hence increase the performance and security of the whole system.

On the other hand, the implementation of the system in a Local Area Network(LAN) can protect the system. Therefore, the access to the system would then be done by someone whose device is connected to that LAN through a local wireless connection.

\section{ACKNOWLEDGEMENTS}

This work was supported by The Centre of Excellence for ICT in East Africa (CENIT@EA) through the Nelson Mandela African Institution of Science and Technology (NM-AIST) in Arusha, Tanzania, under the Programme of Master's degree in Embedded and Mobile Systems.

\section{REFERENCES}

[1] "The Role of Customers in Marketing | Introduction to Business [Deprecated]." https://courses.lumenlearning.com/wmopen-introbusiness/chapter/the-role-of-customers-in-marketing/ (accessed Jun. 17, 2021).

[2] S. Frolova, "Svetlana Frolova THE ROLE OF ADVERTISING IN PROMOTING A PRODUCT Thesis CENTRIA UNIVERSITY OF APPLIED SCIENCES Degree Programme in Industrial Management,” no. May, 2014.

[3] "Boundless Business.” https://courses.lumenlearning.com/boundless-business/chapter/components-of-an-organization/ (accessed Jun. 17, 2021).

[4] "Billboard - Wikipedia.” https://en.wikipedia.org/wiki/Billboard (accessed Jun. 17, 2021).

[5] A. A. OTUOZE, A.O.*, SURAJUDEEN-BAKINDE, N.T., OJO, E.S., AKINDIYA, D.J., IBIDUN, B.F. AND ADENIYI, “IMPLEMENTATION OF AN SMS AND VOICE-CONTROLLED ELECTRONIC BILLBOARD,” vol. 148, no. 2016, pp. 148-162.

[6] "What Are Indoor and Outdoor Advertising and How Do They Differ? | Dominion." 
https://www.dominionprint.com/what-are-indoor-and-outdoor-advertising-and-how-do-they-differ/ (accessed Jun. 17, 2021).

[7] Y. Murumkar, A. Sharma, S. Uppala, and G. Sanas, "Smart Viewer Counter for Digital Billboards," SSRN Electron. J., 2019, doi: 10.2139/ssrn.3419490.

[8] T. K. Liu, Y. W. Huang, and J. Y. Chung, "Interactive wireless electronic billboard," Conf. Proceeding - IEEE Int. Conf. Networking, Sens. Control, vol. 1, no. April 2004, pp. 553-558, 2004, doi: 10.1109/icnsc.2004.1297499.

[9] S. Nigam, S. Asthana, and P. Gupta, "IoT based intelligent billboard using data mining," 2016 1st Int. Conf. Innov. Challenges Cyber Secur. ICICCS 2016, no. Iciccs 2016, pp. 107-110, 2016, doi: 10.1109/ICICCS.2016.7542329.

[10] S. Khan, J. Sallar, S. A. Ali, S. Tuaha, and A. Shariq, "WSN Based Smart Advertisement in Intelligent Transportation System using Raspberry Pi,” 3C Tecnol. innovación Apl. a la pyme, no. May, pp. 486-497, 2019, doi: 10.17993/3ctecno.2019.specialissue2.486-497.

[11] P. Jadhav, A. Deshmane, P. Araj, and R. Sathe, "Smart Billboard System using Data Mining and IOT,” 2020.

[12] C. O. Driscoll, “Advertising with Dynamic Pricing Master in Computer Science,” no. May, 2019.

[13] D. Pandey, A. Ansari, and T. J. Soon, "Three QR Code Related papers T he Effect ive QR Code Development using VB.NET Manish Mat huria Quick Response Code and IT S Use in Libraries: A Recent Trend by Aslam Ansari and Mohd Nazim."

[14] “• Smartphone users 2020 | Statista.” https:/www.statista.com/statistics/330695/number-of-smartphone-users-worldwide/ (accessed Jun. 17, 2021).

[15] "Power Supply - Raspberry Pi Documentation." https://www.raspberrypi.org/documentation/hardware/raspberrypi/power/README.md (accessed Jun. 19, 2021).

[16] B. S. Saracogl@msu.edu, “A Guide to IR/PIR Sensor Set-Up and Testing Instructions, Limitations and Sample Applications."

[17] “PIR Motion sensor module - Seeed Studio.” https://www.seeedstudio.com/PIR-Motion-sensor-module-p-74.html (accessed Jun. 17, 2021).

[18] “HC-SR501 PIR Motion Sensor Module - Elektor.” https://www.elektor.com/hc-sr501-pir-motion-sensor-module (accessed Jun. 19, 2021).

[19] “Overview | PIR Motion Sensor | Adafruit Learning System.” https://learn.adafruit.com/pir-passive-infrared-proximitymotion-sensor (accessed Jun. 19, 2021).

[20] AppsGeyser, “AppsGeyser: Free App Creator \& App Maker. Create Android Apps No Code.” https://appsgeyser.com/ (accessed Jun. 17, 2021).

[21] A. Kurniawan, "Programming on Raspbian OS," in Raspbian OS Programming with the Raspberry Pi, Apress, 2019, pp. 79-96.

[22] I. Volume, "IJMC Volume 5, 2020," vol. 5, no. c, pp. 450-474, 2020.

[23] H. Rachwał, "Outdoor Advertising as an Element Strengthening the Recruitment Campaigns of Universities," Mark. Sci. Res. Organ., vol. 32, no. 2, pp. 27-50, 2019, doi: 10.2478/minib-2019-0029.

[24] G. MAYRA, "Design and Implementation of Motion Detection Alarm and Security System," J. Chem. Inf. Model., vol. 53, no. 9, pp. 1689-1699, 2013.

\section{AUTHORS' PROFILE}

1. Yvonne Iradukunda is a Msc candidate in Embedded and Mobile Systems, specializing in Embedded Systems at Nelson Mandela African Institution of Sciences and Technology. She has a Bachelor Degree in Computer Science with Education obtained from University of Technology and Arts of Byumba-Rwanda. Email : iradukunday@nm-aist.ac.tz

2. Innocent Ciza is a Msc candidate in Embedded and Mobile Systems, specializing in Embedded Systems at Nelson Mandela African Institution of Sciences and Technology. He has a Bachelor Degree in Information and Communication Technologies in the Specialty of Computer Engineering which he graduated from the University of Burundi in Bujumbura-Burundi. His interest lies in Information and Communication Technologies applied in the Education, Business and Healthcare sectors.Email : cizai@nm-aist.ac.tzor cizainno100@gmail.com

3. Salama Ndayisaba is a Msc candidate in Embedded and Mobile Systems, specializing in Embedded Systems at The Nelson Mandela African Institution of Sciences and Technology. He has a Bachelor Degree in Computer Science from Hope Africa University in Bujumbura-Burundi.Email : ndayisabas@nm-aist.ac.tz 
4. Devotha Nyambo is the lecturer at the Nelson Mandela African Institution of Science and Technology. Being a computer scientist, she has developed a great interest in data aggregation, analysis and modeling (statistical and computational). She has worked on various projects internal and external as a researcher focused on tools development, deployment and data management. Her technical skills lie on: use of machine learning models to extract knowledge from data, real world modelling and abstraction. She is also experienced in paperless data capture (tools development, data management and field data collection). She is also experienced in paperless data capture (tools development, data management and field data collection). Email : devotha.nyambo@nm-aist.ac.tz

5. Kisangiri Francis Michael has been working with NM-AIST as a lecturer then Senior lecturer since December 2011. Before joining NM-AIST he worked with Dar-es-Salaam Institute of Technology DIT.He is PhD holder in the field of Telecommunications which he graduated from the Wroclaw University of Technology - Poland in 2008. As academician, he has successfully supervised dozens of researches in the level of MSc and PhD in the category of information science (Artificial intelligence, Epidemic control, Agriculture, Mobile apps, ...) and Wireless communication ( IoT, antenna design, mobile optimization, ...). He possesses good knowledge in Mobile and Wireless communication systems and Artificial intelligence. His research interest is in Machine learning, IoT and MIMO antenna. Email : kisangiri.michael@nm-aist.ac.tz 